Fermilab-TM-2120

\title{
Beam Sweeping System
}

\author{
F.M. Bieniosek*, J. Bielicki† \\ Fermi National Accelerator Laboratory, Batavia, IL 60510 USA \\ A. Cherepakhin, O. Kurnaev \\ IHEP, Protvino, Russia \\ J. Dinkel \\ Creative Designs, Inc., Oak Brook, IL 60523 USA
}

July 24, 2000

\begin{abstract}
This article describes a system for rapidly sweeping a high-energy particle beam in a circular path on a target. The sweeping system deflects the beam in a single-turn rotating-field magnet that combines deflection in both planes into a single unit. The magnet current is up to $10 \mathrm{kA}$ in amplitude and the sweep time is $1.6 \mu \mathrm{s}$. The magnet consists of 4 conductors twisted to provide a uniform line-integral deflecting magnetic field, arranged inside a pressed-powder magnetic core. The pulsed power supply provides the current to the high radiation area of the target vault through several meters of stripline and coaxial cable by means of a magnetic pulse compression circuit based on saturating Ni-Fe and Metglas tape cores. At the Fermilab Antiproton Source increasing proton beam intensities incident on the antiproton production target threaten to deliver energy densities sufficient to locally melt the target in a single pulse. The purpose of the sweep magnet is to spread the hot spot on the target with a sweep radius of up to $0.5 \mathrm{~mm}$, greatly reducing the peak energy deposition.
\end{abstract}




\section{INTRODUCTION}

At Fermilab, antiprotons are collected from the interaction of a $120-\mathrm{GeV}$ proton beam with a solid nickel target. The Main Injector production cycle has a repetition rate of 1.5 seconds, and a beam pulse length of $1.6 \mu \mathrm{s}$. The efficiency of collecting antiprotons from the target rises as the size of the proton beam spot on the target is reduced. However at the same time the peak energy deposition on target rises. Under Main Injector conditions $\left(5 \times 10^{12}\right.$ protons in a 1.6- $\mu$ s pulse), the spot size will have to be increased to at least $0.25 \mathrm{~mm}$ to keep peak energy deposition near current levels. To bring the density of energy deposition with a 0.1 $\mathrm{mm}$ spot size down to currently-existing levels, a system to sweep the beam spot on the target [Ref. 1] has been developed. Several upgrades have been proposed for the Main Injector as part of the Tev33 project [Ref. 2]. These upgrades, e.g., "slip stacking", will result in increases in proton intensity of factors of 2 to 4 above the initial intensity of $5 \times 10^{12}$ protons per pulse on target in the antiproton production process. Under these conditions sweeping becomes increasingly important, and larger sweep radii are required to restore the antiproton yield to the level achievable at low intensity.

Figure 1 shows a layout of the upgraded target station. The upstream sweep magnets will be installed at the end of the AP1 beamline tunnel (after quadrupole magnet PQ9B). The AP1 beamline transports and focuses the $120-\mathrm{GeV}$ protons from the Main Injector onto the target. A pair of upstream sweep magnets will sweep the $120-\mathrm{GeV}$ proton beam. This location is the focal point of the proton lithium lens, which may be necessary to focus the $120-\mathrm{GeV}$ proton beam on target. Antiprotons created in the target are collected by a lithium lens, and deflected by the pulsed magnet into the AP2 beam line for injection into the Debuncher. A single downstream sweep magnet placed in a double module between the collection lens and the pulsed magnet, near the focal point of the collection lens, will redirect the $8 \mathrm{GeV}$ antiprotons exiting the collection lens parallel to the AP2 beamline.

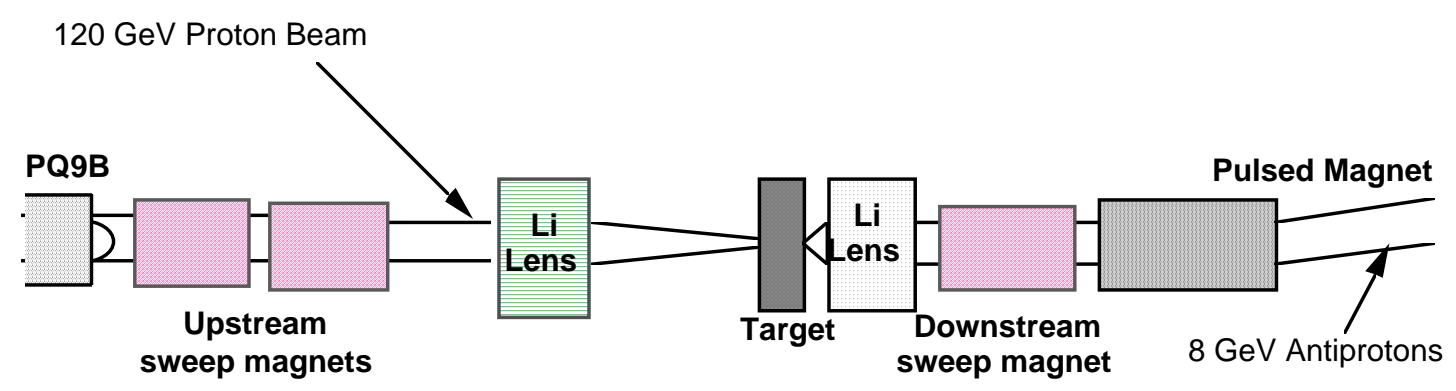

Figure 1. Major components in the target vault of the upgraded target station. Not shown are the pretarget SEM and the beam dump. 


\section{HIGH INTENSITY TARGETRY}

Measurements of yield with beam spot position on the target show a Gaussian-shaped yield curve $Y(x), Y(y)$, in both horizontal and vertical dimensions, with $\sigma_{x}=0.48 \mathrm{~mm}$, and $\sigma_{y}=$ $0.65 \mathrm{~mm}$. The shape of the yield distribution is determined by the acceptance and lattice functions of the Debuncher and the AP2 beam line. If the incoming proton beam is also a Gaussian, $\sqrt{2 / \pi} \sigma_{b x}^{-1} \exp \left(-x^{2} / 2 \sigma_{b x}^{2}\right)$ in both planes, with characteristic spot size $\sigma_{b x}$ and $\sigma_{b y}$, then the dependence of the yield on spot size, normalized to the yield for an infinitesimal point beam is

$$
Y=\int Y(x) I(x) Y(y) I(y) d x d y
$$

which integrates to

$$
Y=\frac{\sigma_{x} \sigma_{y}}{\sqrt{\sigma_{x}^{2}+\sigma_{b_{x}}^{2}} \sqrt{\sigma_{y}^{2}+\sigma_{b_{y}}^{2}}} .
$$

This curve is plotted in Fig. 2 for a circular beam spot. Also shown are MARS10 calculations of energy deposition in a copper target as a function of beam size for $\mathrm{N}=5 \times 10^{12}$ protons per pulse.[3] A small fraction (typically less than 10\%) of the deposited energy is released as a stress wave by the elastic properties of the metal; the remainder is deposited locally for a time much longer than a beam pulse. The spot size under current operating conditions is about $\sigma_{b x}=0.15 \mathrm{~mm} ; \sigma_{b y}=0.23 \mathrm{~mm}$. Estimates of the peak instantaneous energy deposition for the highest intensity achieved to date $\left(3.4 \times 10^{12}\right)$ indicate an energy deposition of about $800 \mathrm{~J} / \mathrm{g}$. This is above the melting point of copper (about $600 \mathrm{~J} / \mathrm{g}$ ), and close to the melting point of nickel (about $1000 \mathrm{~J} / \mathrm{g}$ ). Local disintegration of the target has been observed when the target rotation mechanism failed. The damage was caused by the combination of the integrated radiation dosage to the affected spot, and the repetitive thermal and mechanical stresses. When the target was slowly rotated to regularly expose fresh material to the beam, less severe damage was observed. In order to maintain peak energy deposition below present levels after the Main Injector begins operation, it will be necessary to increase the spot size. The alternative is to sweep the beam on the target, and reduce the spot size to the smallest attainable, leading to a $15-20 \%$ increase in yield.

The effective size of the energy deposition profile is comparable to or larger than the beam spot size. For a circular Gaussian energy deposition profile with equal variance $\sigma$ in both planes, swept in a circle of radius $r_{0}$, the radial energy deposition profile can be shown to be

$$
E(r)=\exp \left[-\frac{r^{2}+r_{0}^{2}}{2 \sigma^{2}}\right] I_{0}\left(\frac{r r_{0}}{\sigma^{2}}\right)
$$

where $I_{0}$ is the modified Bessel function. The energy deposition curve (Eq. 2) is shown in Fig. 3 for no sweep, and two ratios of sweep radius to the energy deposition spot size 
$\sigma=\sigma_{\mathrm{h}}$. Increasing amplitude of beam sweep rapidly reduces peak energy deposition for $r_{0} / \sigma_{h}$ near 2. The effect is that a sweep radius of $0.33 \mathrm{~mm}$ reduces the peak energy deposition to about $600 \mathrm{~J} / \mathrm{g}$ for $5 \times 10^{12}$ protons per pulse[3]. This level of energy deposition is likely to be acceptable for reliable operation in nickel targets. However, continued increase in sweep radius has a weaker effect on peak energy deposition as the heat deposition profile becomes hollow.

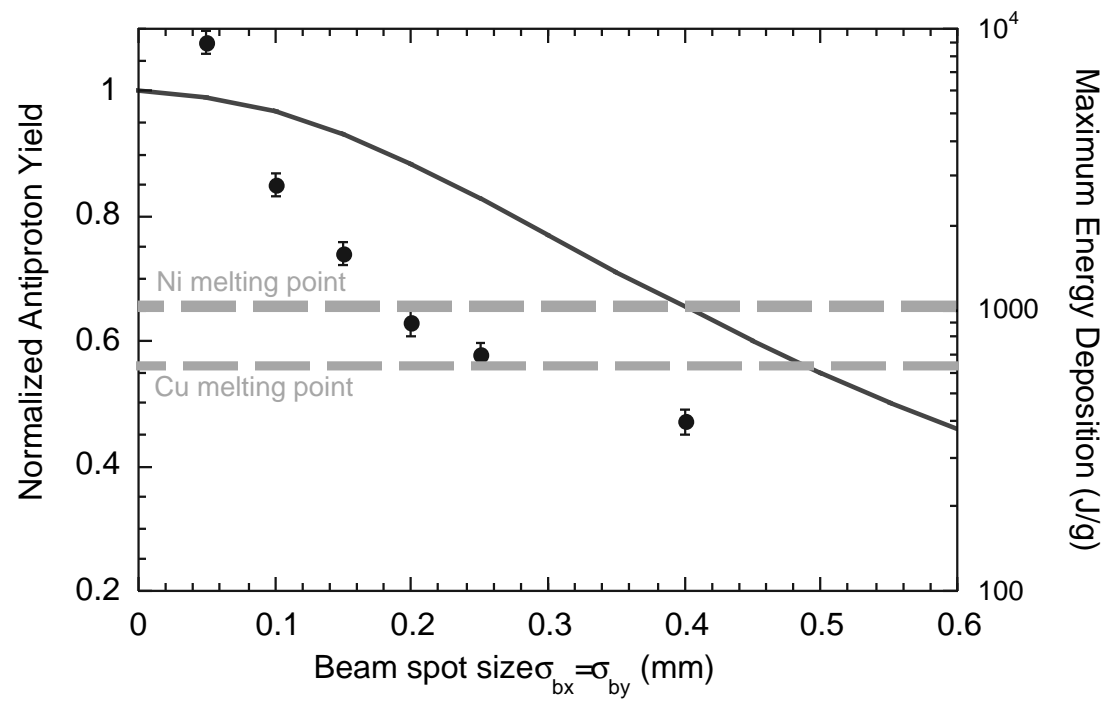

Figure 2. Scaling of yield (curve) and peak energy deposition (points with error bars) in the target for proton beam intensity of $5 \times 10^{12}$ as a function of beam spot size. The values for energy deposition were taken from Ref. 3.

The calculations of particle distribution and yield presented in Figures 4 through 7 below were performed with the program MCLENS[Ref. 4]. MCLENS does a Monte-Carlo simulation of particles emanating from the target, and follows them to the acceptance aperture of the downstream AP2 beam line and Debuncher ring. It is based on previous work [Ref. 5] on antiproton particle distributions and secondary production, but contains a realistic lens model to allow direct comparison with experimental data without a forced assumption of a uniform lens gradient. Aberrations in the lens strongly influence the shape of the yield curves. The program also allows the calculation of particle distributions radially outside of the lens. It is used to model effects of varying target station geometries, beam sweeping, AP2 and Debuncher aperture, etc. Assumptions used in the calculations (except as noted otherwise) include an upgraded AP2/Debuncher (32 $\pi \mathrm{mm}$-mrad acceptance, matched at the target), $2000 \mathrm{~V}$ lens voltage, and a $0.15-\mathrm{mm}$ spot size. 


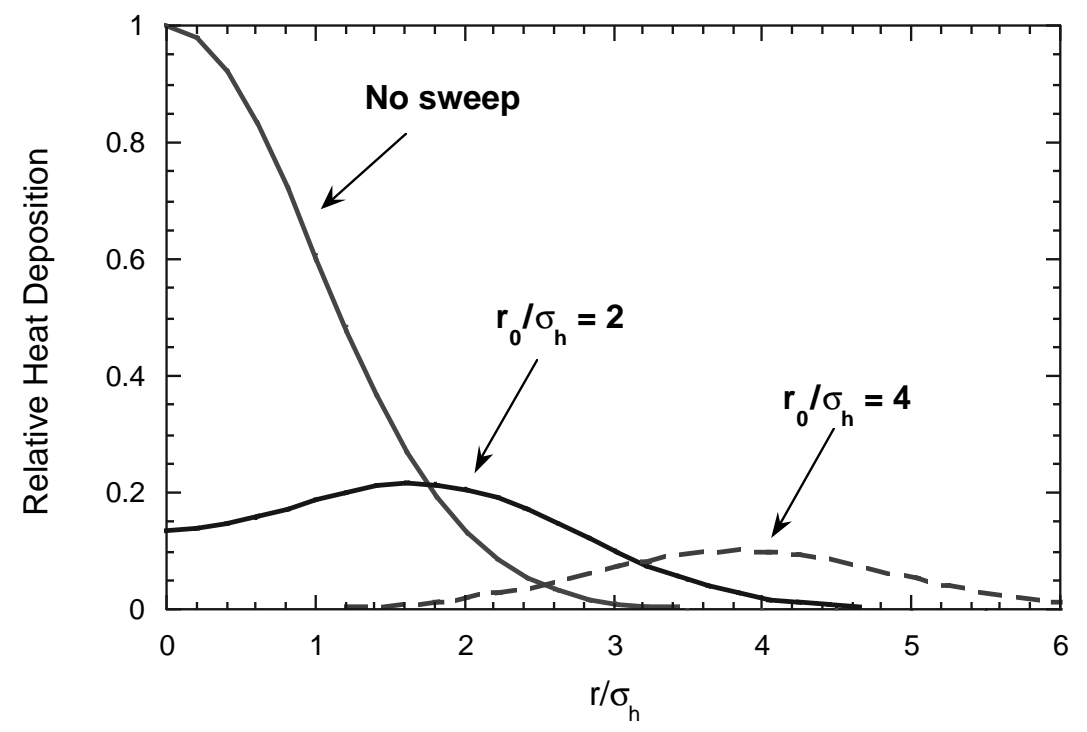

Figure 3. Effect of beam sweeping on the local energy deposition profile for an initially Gaussian energy deposition. Curves are for no sweep, and two ratios of sweep radius to the spot size $\sigma_{\mathrm{h}}$ of the energy deposition profile.

In the absence of beam sweeping, the yield degrades as the intensity increases because the beam spot size must be increased. Sweeping allows reduction in spot size and restoration of yield. As the beam intensity increases, sweeping becomes increasingly important, and the sweep radius required to completely restore yield to a given level increases. Fig. 4 shows estimates of yield for several sweep radii and beam intensities, assuming a circular sweep, and varying spot size to maintain a fixed energy deposition of $800 \mathrm{~J} / \mathrm{g}$. These calculations show the much greater importance to be attached to the sweeping system as beam intensity on target increases beyond that proposed for initial Main Injector operation. The potential effect of the sweeping system is to increase the yield by about $15 \%$ at $5 \times 10^{12}$ (at $0.33-\mathrm{mm}$ sweep radius), $50 \%$ at $1 \times 10^{13}$ (at $0.5 \mathrm{~mm}$ sweep radius), and $100 \%$ at $2 \times 10^{13}$ (at $0.75 \mathrm{~mm}$ sweep radius).

A histogram of the radial distribution of collected particles at the lens and the sweep magnet is plotted in Figure 5. Sweeping the beam has no significant effect on the distribution. The assumptions represent operation with optimal lens timing (relative phase difference between 


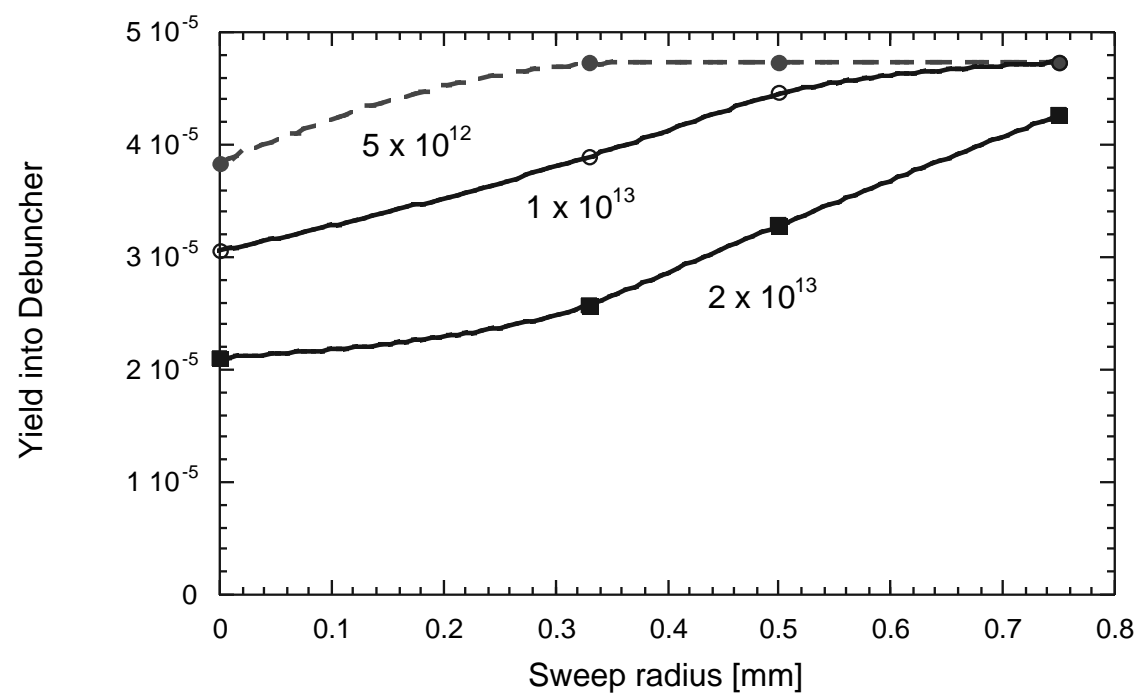

Figure 4. Yield vs. sweep radius at three incident proton intensities. Beam spot size is adjusted to fix energy deposition at $800 \mathrm{~J} / \mathrm{g}$. Lens voltage is $2400 \mathrm{~V}$.

the beginning of the lens current pulse and the time of the beam pulse), and a relatively large AP2/Debuncher transverse acceptance aperture of $32 \pi \mathrm{mm}$-mrad. (As the acceptance aperture increases, the distribution of acceptance becomes wider.) Note that a modest fraction of the collected particles $(10 \%$ or less) exit the lens outside of the current-carrying lithium core of the lens $(10 \mathrm{~mm})$. The calculation includes scattering and absorption in the titanium shell surrounding the lithium, but does not include the effect of a steel collar at the downstream end of the lens, which has an inner radius of $11 \mathrm{~mm}$. This collar essentially eliminates the particles beyond $11 \mathrm{~mm}$. Therefore the sweep magnet needs to be designed to accept particles up to a radius of about $11 \mathrm{~mm}$, although the great majority of particles are well inside this radius.

The effect of beam sweep on yield and field uniformity requirements are calculated in Figure 6. For example, the curve labeled 0.33 represents the yield as a function of downstream sweep angle for a fixed upstream sweep radius of $0.33 \mathrm{~mm}$. At the optimal sweep angle (about $1.5 \mathrm{mrad}$ ) the yield is restored to its level for no sweep. Note that even in the absence of a downstream sweep, the yield is only reduced by $15 \%$. This indicates that the beam is relatively insensitive to field uniformity -- a 100\% field error in this case reduces yield by only 15\%. Field uniformity becomes more important as the sweep radius increases. 


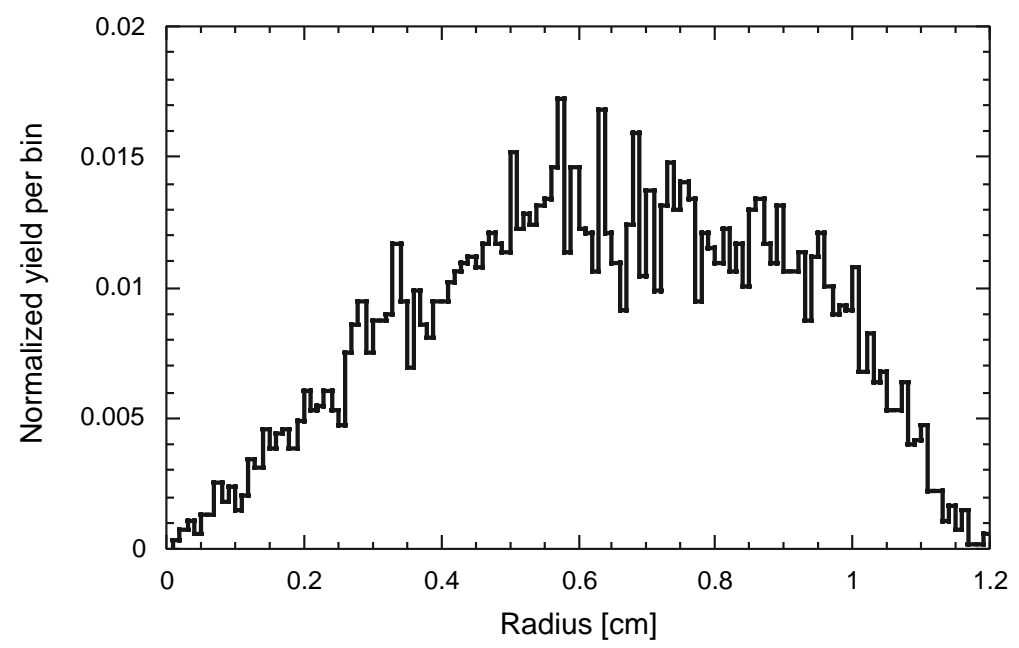

Figure 5. Radial distribution of collected antiprotons at the downstream sweep magnet. The lens phase is 0.65 . The outer radius of the lithium portion of the collection lens is $1 \mathrm{~cm}$. The total number of antiprotons collected is 5465 .

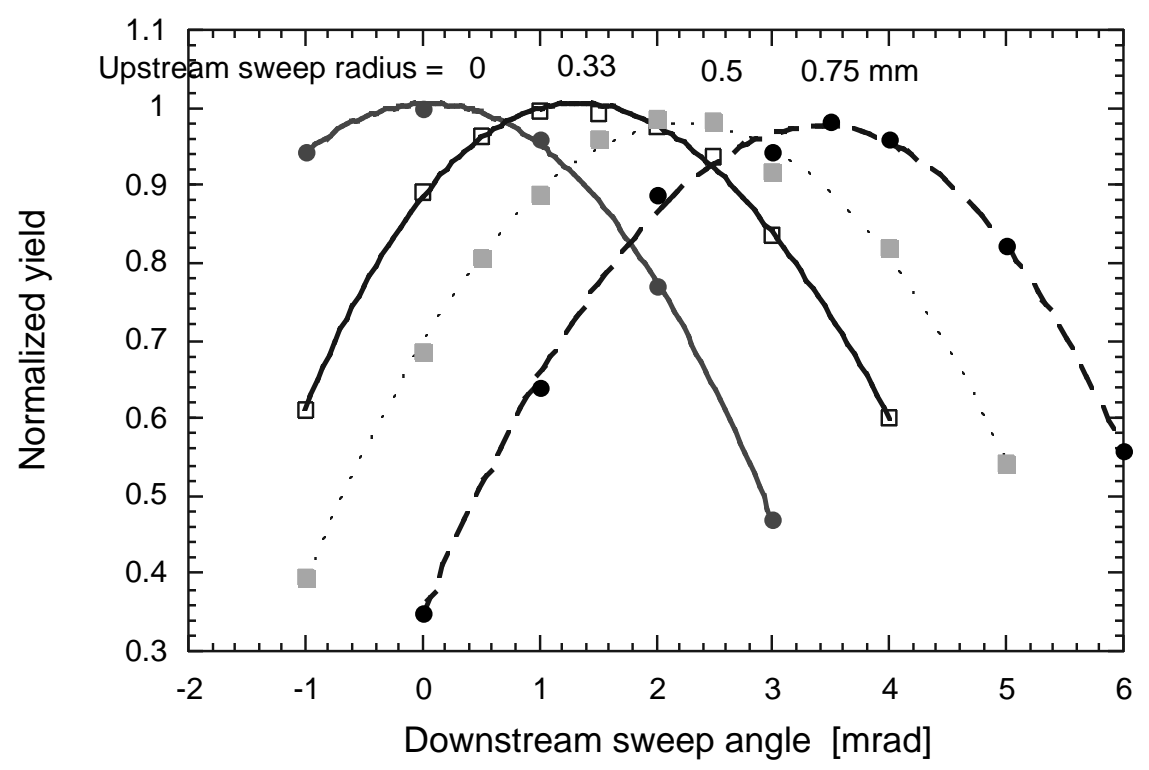

Figure 6. Yield as a function of downstream sweep angle for several sweep radii. 


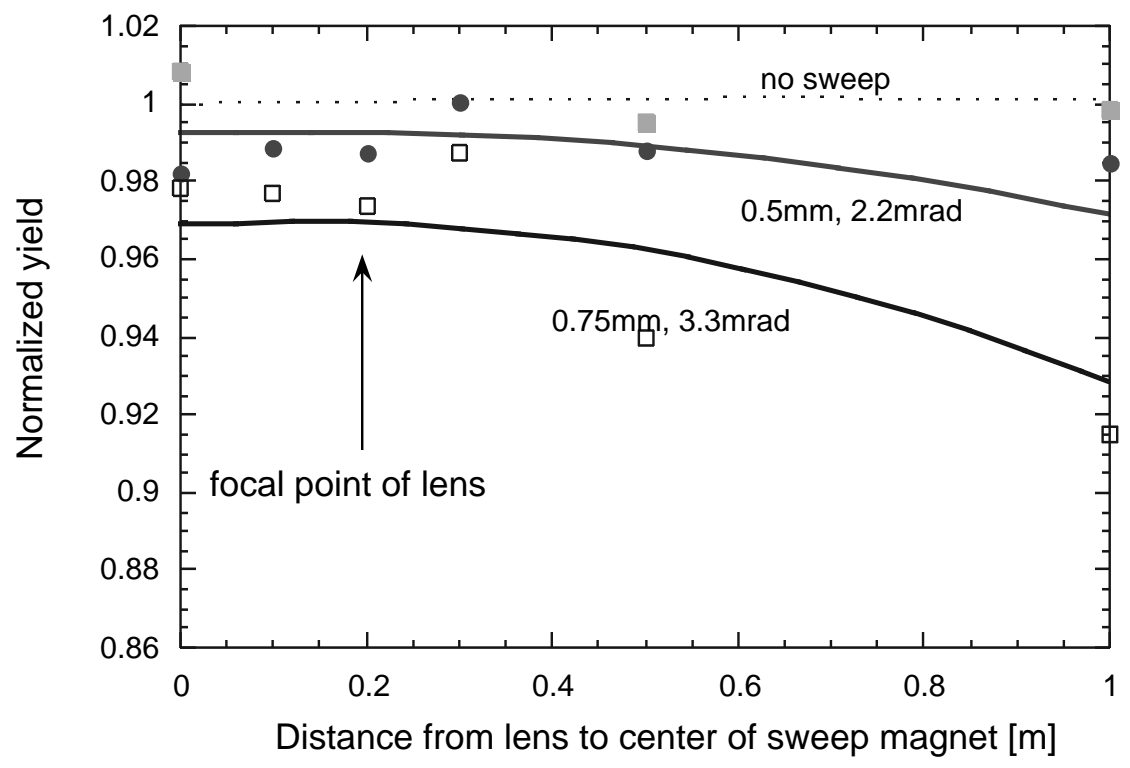

Figure 7. Yield as a function of axial position of downstream sweep magnet.

Finally, the dependence of yield on axial placement of the sweep magnet is calculated in Figure 7 . The plot shows that the yield is relatively insensitive to axial position over a wide range. This fact gives us freedom to place the sweep magnet axially in any convenient location near the lens. It shows a very small $(1 \%)$ loss in yield due to sweeping for the maximum design sweep radius $(0.5 \mathrm{~mm})$. The loss in yield rises to roughly $3 \%$ for $0.75 \mathrm{~mm}$.

\section{HIGH VOLTAGE TEST}

Ionization of the air by the particle shower downstream of the target increases the conductivity of the air between the conductors. Electrical losses through the ionized-air path across the gap reduce the $\mathrm{Q}$ of the circuit driving the magnet. A dummy test module was installed to measure the leakage current between the two conductors symmetrically placed parallel to the beam path. Both measurements and estimates based on CASIM calculations indicated an current drain on the order of $100 \mathrm{~A}$, an acceptable amount. The measured leakage current for two different beam intensities is shown in Figure 8. The current through the air gap turned on rapidly at the beginning of the beam pulse and the gap rapidly opened after the end of the beam pulse. The ringing in measured current after the end of the beam pulse is the ringing of the cable between the current source and the test module. Avalanche ionization of the air is not 


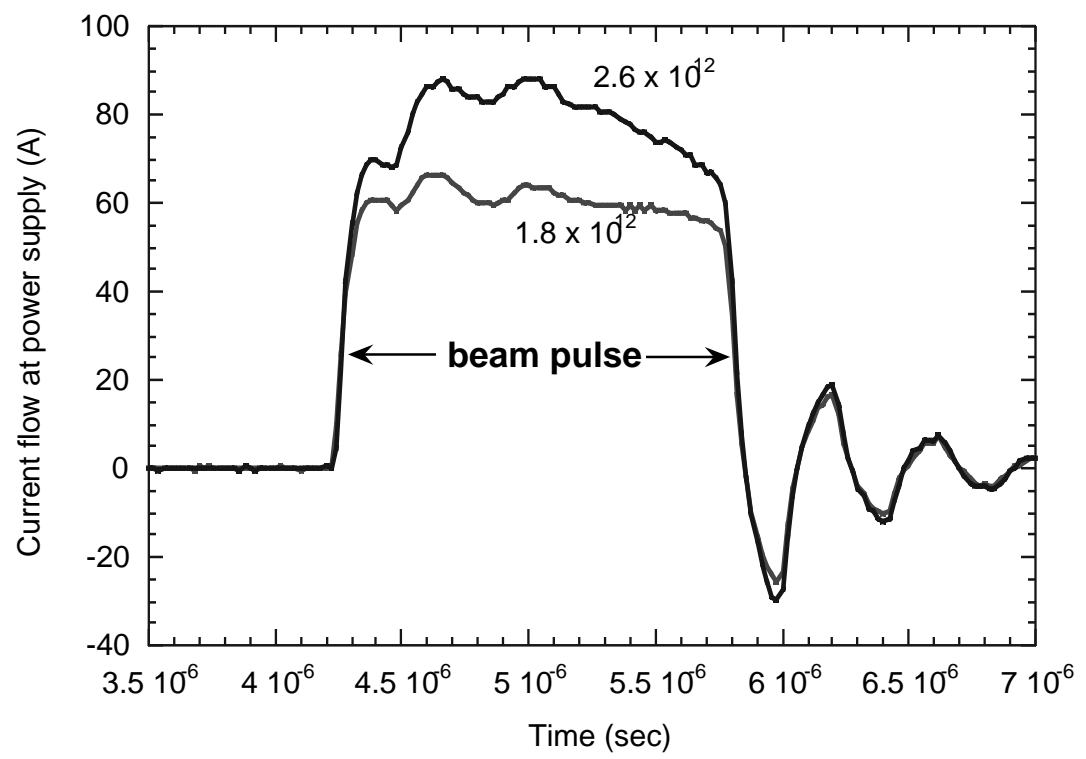

Figure 8. Ionization current during beam pulse through a pair of conductor plates $3-\mathrm{cm}$ wide $\times 3-\mathrm{cm}$ gap $\mathrm{x} 24-\mathrm{cm}$ length at the location of the sweep magnet. The voltage across the gap was $10 \mathrm{kV}$ and measurements were taken at the two beam intensities indicated.

a problem, as long as peak electric fields are kept well below the breakdown level, i.e. $\mathrm{E}<10$ $\mathrm{kV} / \mathrm{cm}$. The test module was instrumented to provide information on heat dissipated and short-term magnetic effects in ferrite and tape-wound cores caused by the particle shower downstream of the target. Ref. 6 describes the test in detail.

\section{SWEEP MAGNET}

A rotating beam spot on the target can be created by two kickers, one vertical and one horizontal. The excitation currents should be sine waves in quadrature. The approach we have chosen is to combine the kickers into the same magnet which has two pairs of conductors placed 90 degrees apart inside a circular magnet yoke.

Three identical magnets will be used. A pair of upstream magnets, placed at the downstream end of the AP1 beamline where the toroid M:TOR109 now resides, will sweep the 120-GeV proton beam. This location is near the focal point of the proton lithium lens, which may be used to focus $120-\mathrm{GeV}$ proton beam on target. A single downstream magnet placed in a double module between the collection lens and the pulsed magnet will redirect the $8 \mathrm{GeV}$ antiprotons exiting the collection lens parallel to the AP2 beamline. The sweeping radius is much smaller than the $2 \mathrm{~cm}$ diameter of the lithium collection lens and the aperture of the AP2 beam line. 
The beam sweeping system is designed to trace a circular pattern on the target over the 1.6$\mu$ s proton beam pulse with a nominal radius of $0.33 \mathrm{~mm}$ and a maximum radius of $0.5 \mathrm{~mm}$. The magnet has a circular aperture of $28.5 \mathrm{~mm}$. The portion of the antiproton flux that is focused by the lithium lens and collected downstream has a diameter of $22 \mathrm{~mm}$. The physical length of the magnet is $56 \mathrm{~cm}$. Two of the sweep magnets will be installed upstream of the target, and one sweep magnet downstream of the target. The upstream deflection required to deliver the $0.5 \mathrm{~mm}$ sweep is $0.17 \mathrm{mrad}$ in the absence of the proton lens, and $0.28 \mathrm{mrad}$ if a lithium lens is required to provide final focus of the proton beam. The downstream deflection required to center the antiprotons in the collection beamline is $2.2 \mathrm{mrad}$, as shown in Figure 6. Assuming an effective magnetic length of $52 \mathrm{~cm}$, for two upstream magnets the field on axis is 0.66 and $1.09 \mathrm{kG}$, respectively, without and with the presence of a proton lens. For a single downstream magnet the average field on axis is 1.26 kGauss.

System requirements on timing jitter, field regulation, and field uniformity may be estimated from the effective RMS radius $x_{R M S}$ of a displaced beam spot at the target

$$
x_{R M S}=\left(\sigma_{x}^{2}+\Delta x^{2}\right)^{1 / 2}
$$

where $\sigma_{x}$ and $\Delta x$ are the Gaussian spot size and the displacement in a given direction. If we wish to limit the increase in $x_{R M S}$ to less than $10 \%$, the basic criterion becomes $\Delta x / \sigma_{x}<$ 0.46 , or typically, $\Delta x<0.05 \mathrm{~mm}$.

Timing jitter/sweep time. Timing jitter between horizontal and vertical power supplies causes a deviation of the beam path from a perfect circle. Timing jitter between upstream and downstream magnet pairs causes a lag or lead of the magnet partners with respect to each other as they trace out their circular paths. The distortions created by these errors are

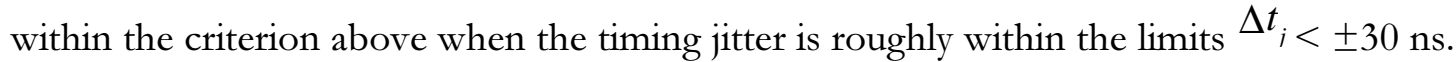
In addition, the magnet sweeps are also restricted to agree within $\pm 30 \mathrm{~ns}$, out of $1.6 \mu \mathrm{s}$.

Field uniformity/regulation. If we again limit the distance the upstream and downstream paths are allowed to be displaced with respect to each other, the requirement becomes $\Delta B / B_{0}< \pm 7 \%$ for both field uniformity and pulse-to-pulse field regulation in the sweep magnets.

The magnets have a 2-phase, 4-conductor winding excited by two power supplies that deliver $625 \mathrm{kHz}$ sinusoidal current waveforms in quadrature to generate a $625-\mathrm{kHz}$ rotating dipole field $\left.{ }^{7}\right]$. The magnetic field due to two pairs of current-carrying conductors oriented $90^{\circ}$ apart is rotating on axis if the current in the two pairs of conductors is $I_{0} \cos (\omega t)$ and $I_{0} \sin (\omega t)$. Figure 9 shows the magnetic field lines as calculated by POISSON at four times in the cycle. 

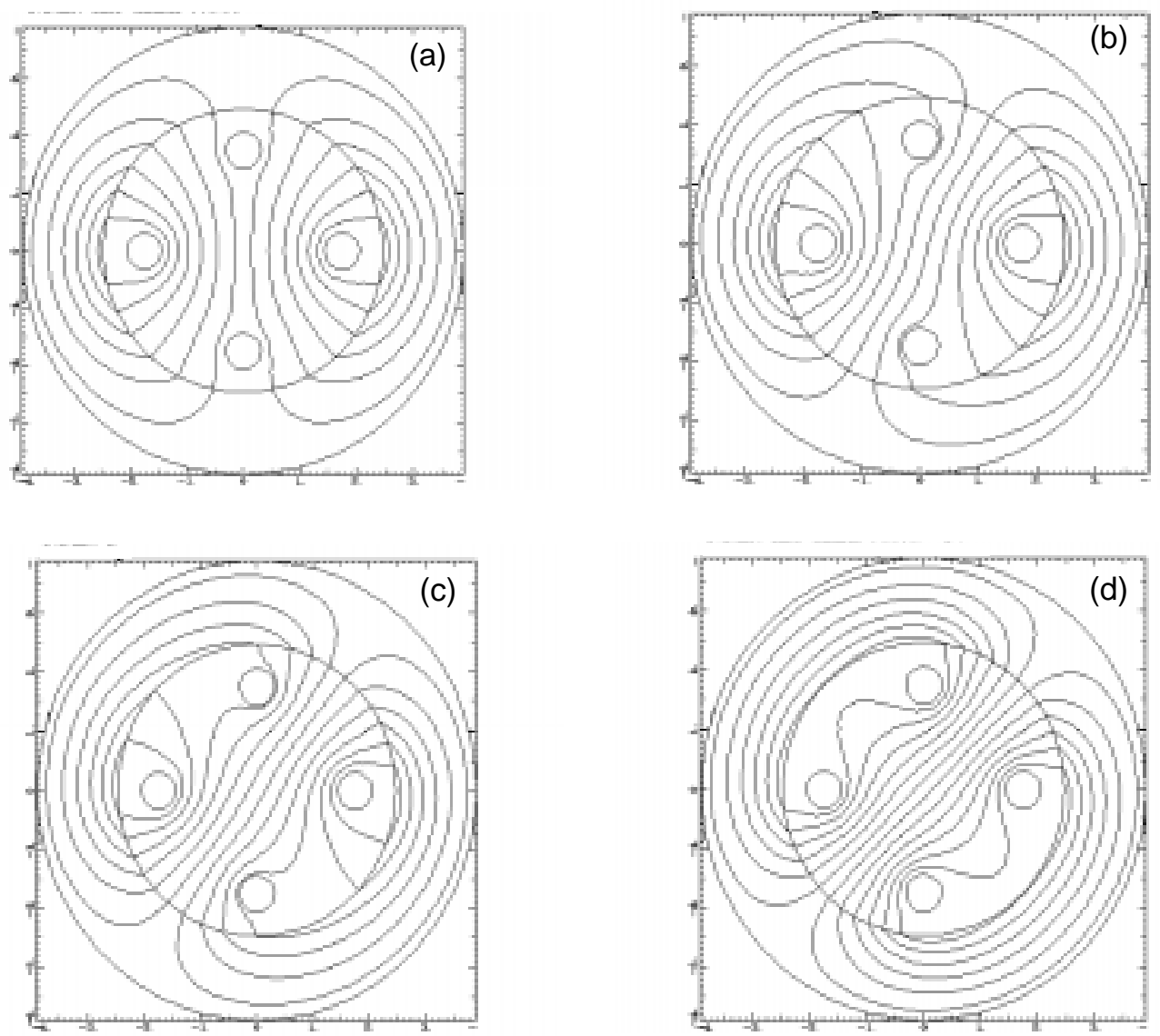

Figure 9. Local magnetic field structure for a 2-phase rotating-field sweep magnet. Current to horizontal pair of conductors has a $\cos (\omega t)$ time dependence; current to vertical pair of conductors has a $\sin (\omega t)$ time dependence. Field distribution is shown for (a) $\omega t=0$, (b) $\omega t=\pi / 12$, (c) $\omega t=\pi / 6$, (d) $\omega t=\pi / 4$. A magnetic core surrounds the conductors. Dimensions are in $\mathrm{cm}$.

The local field uniformity in itself is not sufficient for use as a sweep magnet. However if the conductors are twisted such that the axial current component integrated over the length of the magnet has the distribution $I_{0} \sin (\omega t-\theta)$, the line integral of the field along the beam path is uniform and rotating. The conceptual design for the windings is indicated in Figure 10. The windings are in the form of two single-turn circuits placed symmetrically at 90degree intervals. Each circuit has a twist of 180 degrees over the length of the magnet. The shape of the conductors is $z=\left(z_{0} / 2\right) \sin (\theta)$, where $z_{0}$ is the axial length of the magnet, and $z=0$ is defined as the axial center of the magnet. The external power-supply connections for the two circuits are indicated respectively by the black and shaded circles. The first circuit (black) has a current flowing at the instant pictured, indicated by the arrows, entering the magnet at $\theta=180^{\circ}$ (black circle) and leaving at $0^{\circ}$ (or $360^{\circ}$ ). The current flows from the midplane at the end rings, where it rotates $180^{\circ}$ and returns to the midplane to the opposite terminal of the circuit. As time progresses, the second circuit (gray) begins to carry 
current in the same manner, and the overall current distribution rotates if the current in the two circuits is properly phased. The end rings are common to the two circuits, and, if the magnet is driven by a bipolar power supply, are at ground potential. This magnet design is simple and mechanically robust. There are no breaks in the windings for power supply leads, and since the voltage is nominally zero at the ends, there is no need to allocate space to provide electrical insulation from neighboring devices in the target station.

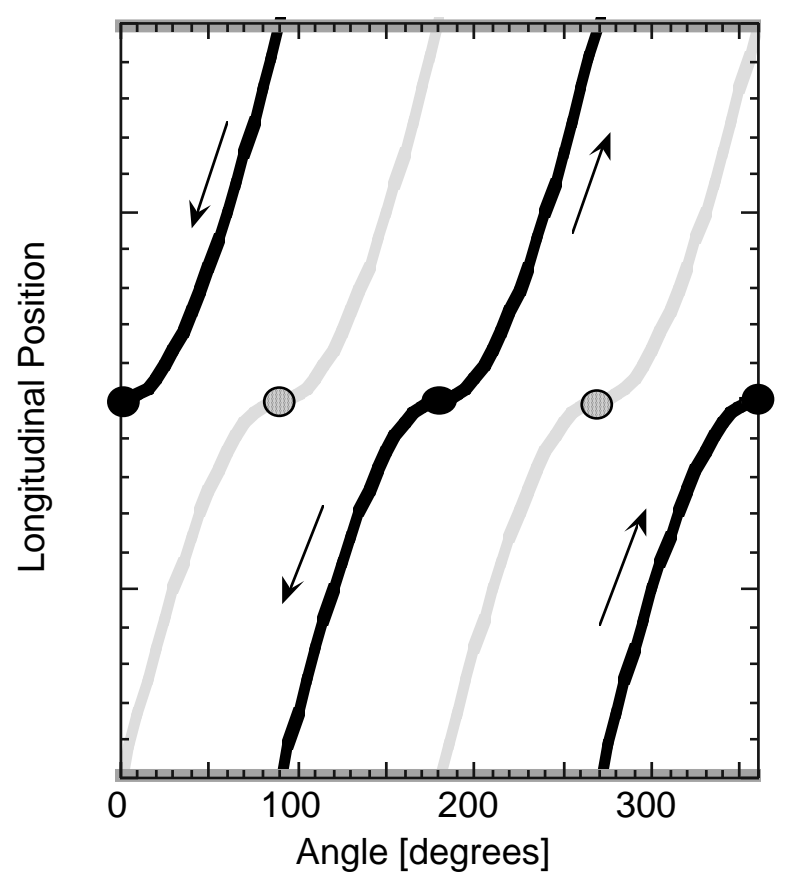

Figure 10. Shape of windings. The black curves represent one circuit, the gray curves represent the orthogonal circuit. Direction of current flow is indicated in one of the circuits.

The average deflecting field requirement is $1.26 \mathrm{kG}$, corresponding to a $0.5-\mathrm{mm}$ sweep radius, on the downstream sweep magnet. Because of the twist in the conductors, the peak field on axis must be a factor $4 / \pi$ times larger than the average field, or $1.60 \mathrm{kG}$. The ratio of the magnet current to the local magnetic field on axis, calculated by POISSON for a straight conductor, is $6.1 \mathrm{~A} /$ Gauss. Thus to provide the desired field, the peak current 
required from the power supply is $9.8 \mathrm{kA}$, and the peak inductive voltage drop across the magnet is $7.7 \mathrm{kV}$ (peak voltage to ground $3.85 \mathrm{kV}$ ) for a magnet inductance of $0.2 \mu \mathrm{H}$.

A cross sectional view of the sweep magnet is shown in Figure 11. The clear bore of the magnet is $28.5 \mathrm{~mm}$. The conductors are hollow 1/4" $(6.35-\mathrm{mm})$ diameter aluminum tubes. Hollow aluminum tubes were chosen to minimize the beam-induced heating of the conductors, and to allow the passage of cooling air through the center of the conductors. A ceramic tube provides electrical insulation between the conductor assembly and the magnetic cores. No vacuum wall is required, because the beam is transported through air from upstream of the target to downstream of the pulsed magnet. Molybdenum Permalloy Powder (MPP) cores were chosen for the sweep magnet. These cores consist of permalloy powder cemented and pressed with an inorganic ceramic-type insulation. They feature a relatively high Curie temperature $\left(\mathrm{T}_{\mathrm{c}}>400^{\circ} \mathrm{C}\right)$ and good thermal conductivity. The cores are press-fit into a water-cooled nickel housing. Nickel was chosen for the pipe material because it has the same thermal expansion coefficient as the MPP core.

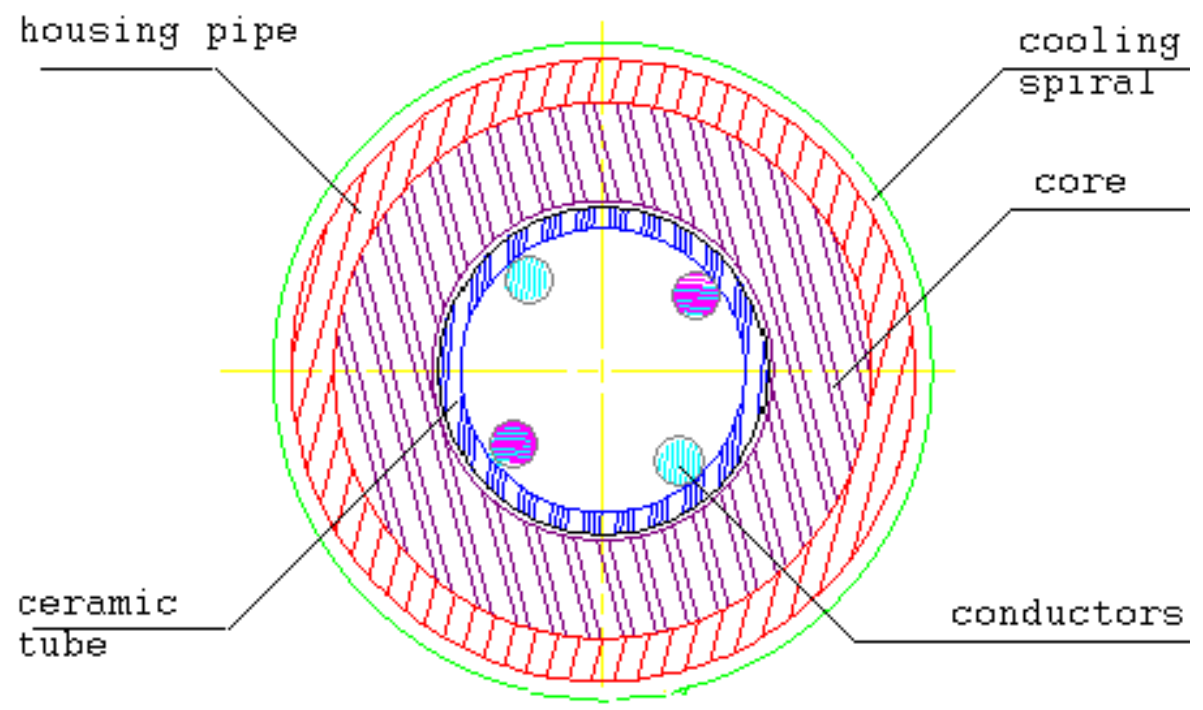

Figure 11. Sweeping magnet cross section.

MARS10 and CASIM calculations of energy deposition by hadron and electromagnetic cascades, supported by measurements on the high-voltage test module, show significant heating of iron and ferrite magnet cores downstream of the target. Total heating increases linearly with particle flux, and is a strong function of the radius of the magnet core. Steadystate temperature rise of the core is determined by thermal conductivity of the material and the rate at which heat is removed at the surface.

Four magnetic materials were considered for use in the magnetic yoke, before choosing the powder core material. 
1. Ferrites. Ferrites have poor thermal conductivity, a low Curie temperature, a low saturated magnetic flux density, and exhibit short-term radiation effects. [Ref. 5] It would be necessary to operate a ferrite-core magnet with a large inner-radius $(3 \mathrm{~cm})$ core and carefully cool the ferrite. Maintaining adequate cooling under these conditions would be very difficult.

2. Silicon-steel lamination. A stack of thin laminations with thickness $100 \mu \mathrm{m}$ or less is a potential candidate for the magnet yoke. Expected voltage per lamination for $100 \mu \mathrm{m}$ laminations is in the range $2-4$ Volts, which may exceed the strength of the insulation. Each magnet requires over 5000 laminations. Difficulties are expected in preparing and stacking these thin laminations.

3. Tape-wound cores. The fact that the magnetic field lines must twist on entering the cores from perpendicular to parallel to the direction of the tapes causes excessive losses in the magnet.

4. Powder cores. There are three candidate powder core materials. These are MPP (2Mo $81 \mathrm{Ni}-17 \mathrm{Fe})$, High-flux $(50 \mathrm{Ni}-50 \mathrm{Fe})$, and Sendust $(85.5 \mathrm{Fe}-9.5 \mathrm{Si}-5 \mathrm{Al})$. All three are commercially available in cores of the desired size, and at low cost. They have relatively low losses, and have adequate inorganic insulation. A magnet assembled with powder cores has the advantages of ease of construction, and the relatively high thermal conductivity and Curie temperature of this material. The fairly small thermal stresses are contained by pressfitting the cores in a nickel housing. The core material chosen is $\mu=60 \mathrm{MPP}$, because this material has the lowest losses. Eddy-current losses in the MPP material increase slightly under intense neutron bombardment[8]. This is not likely to be a major concern, however, since the core is not the dominant loss mechanism in the ringing circuit.

Estimated beam thermal power is 54 Watts per core at $1 \times 10^{13}$ protons per pulse and a 1.5 second repetition rate, or a total power of 1944 Watts for 36 cores. This amount of heat can be removed in a water cooling circuit. The expected temperature drop from core ID to OD is about 5 degrees Centigrade.

A longitudinal view of the magnet is shown in Figure 12. It can be seen from Figure 12 that the current leads are located in the midplane of the magnet. The conductors are grounded at the end ring by the end flange on both sides of the magnet. Four ceramic bushings are used to support the current leads and insulate them from the magnet body. Spiral fingerstock is attached to copper spacers located between each two pairs of cores. It serves as a slide guide and stress relief for the insulating ceramic 


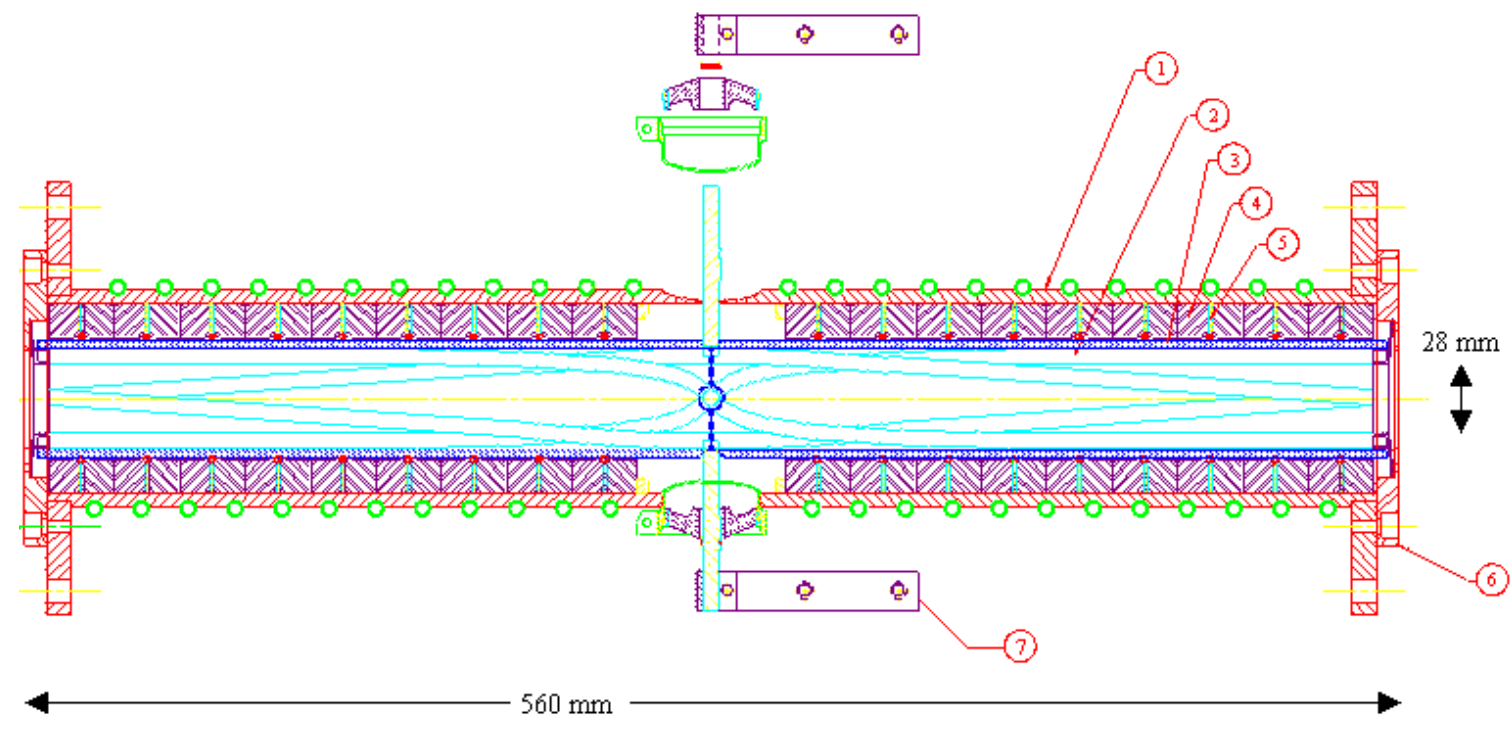

Figure 12. Longitudinal cross section of rotating field magnet. 1 - housing pipe, 2 twisted conductors, 3 - ceramic pipe, 4 - MPP core, 5 - spacer, 6 - end flange, 7 - current lead tube, and provides a thermal conductive path for removal of beam-induced heating from the ceramic tube and the current conductors.



Horizontal position, $\mathrm{mm}$

Figure 13. Line-integrated magnetic field distribution in the aperture of the rotating field magnet. $\mathbf{0}$ in horizontal scale corresponds to the vertical magnet axis. Horizontal scale is $\mathrm{mm}$. Curve 1 is the field distribution in median plane. Curve 2 is measured at $+7 \mathrm{~mm}$ vertically above median plane and curve 3 is measured $-7 \mathrm{~mm}$ (below). Field measurements are normalized to field on axis. 


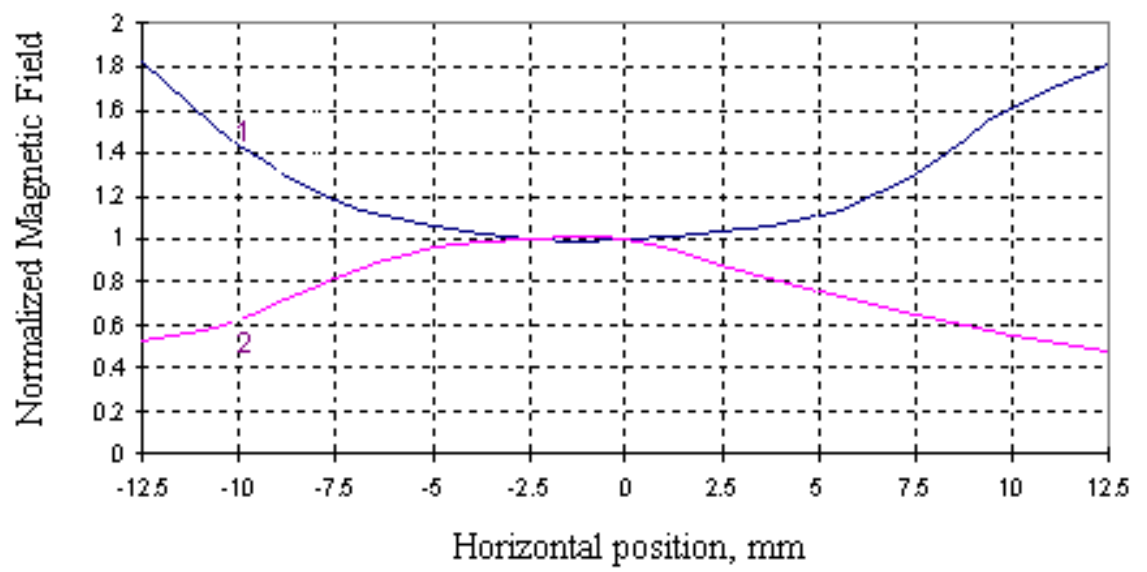

Figure 14. Local magnetic field distribution in the aperture of the rotating field magnet. 0 - corresponds to the magnet vertical axis. Curve 1 represents the field distribution when the measuring coil is $2 \mathrm{~cm}$ inside the magnet end ring. Curve 2 is measured at $20 \mathrm{~cm}$ from the end ring.

Magnetic measurements of the sweep magnet were performed with stretched wires. Ribbon cable was used to pick up the $\mathrm{dB} / \mathrm{dt}$ signal along the whole magnet length and then was integrated to obtain a signal proportional to magnetic flux through the measurement loop. The signal amplitude was as expected. The line-integrated field distribution for three vertical planes is shown in Figure 13. The local field distributions in the median plane measured with a small pickup coil in two longitudinal positions is shown in Figure 14.

\section{POWER SUPPLY}

The sweep magnet must be provided with an oscillating current with amplitude up to $10 \mathrm{kA}$ at $625 \mathrm{kHz}$ from a power supply located on the floor of the AP0 service building. The energy will be supplied through cables over a distance of approximately $10 \mathrm{~m}$ into the target vault, and by $2.5 \mathrm{~m}$ of strip line through steel shield modules to the magnets at the bottom of the target vault. A simplified power supply circuit diagram is depicted in Figure 15. The solid-state power supply is based on a two-stage compression circuit with saturating reactors. Pulse compression facilitates transfer of the current pulse to the ringing circuit, and provides the capability to utilize a SCR switch for resonant charging of the first stage capacitor C1 using a 1:10 step-up transformer. 


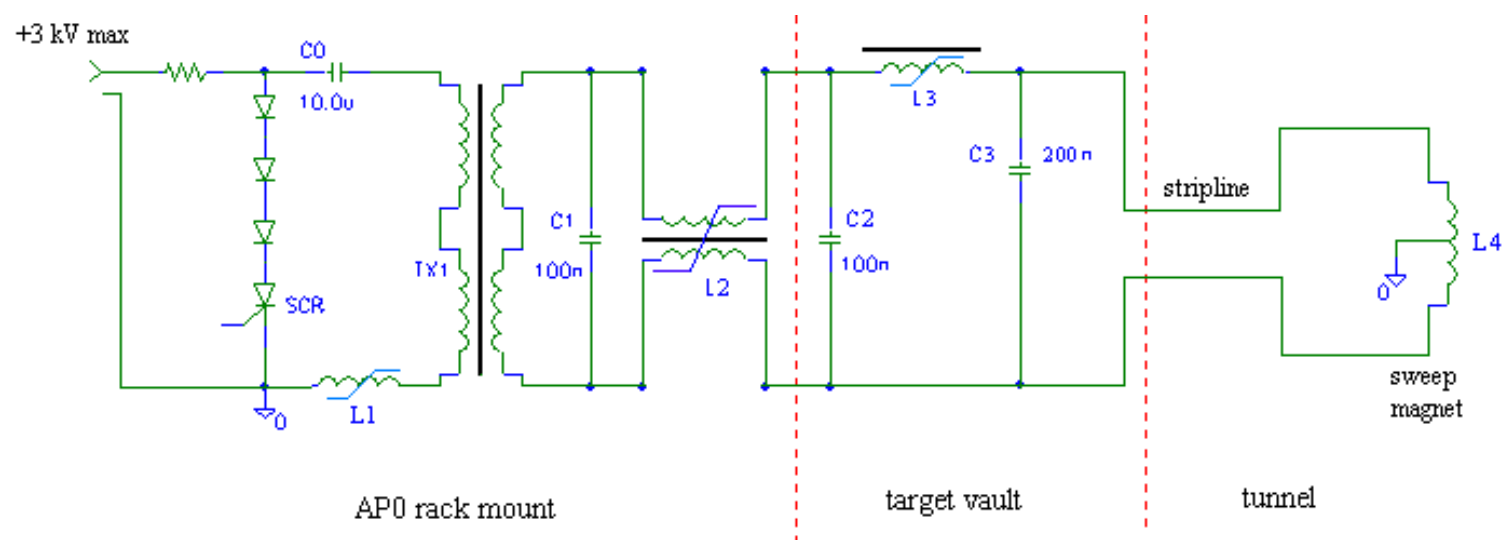

Figure 15. Simplified schematic diagram of power supply.

Energy storage capacitor $\mathrm{C} 0$ is initially charged up to $3 \mathrm{kV}$ by the DC high voltage supply. The energy is transferred to the first compression stage capacitor C1 via the step-up transformer by closing the thyristor switch SCR. The SCR (Westcode) is rated at $3.6 \mathrm{kV}$. The time period for the charge transfer is chosen to be about $15 \mu$ s because of the $\mathrm{dI} / \mathrm{dt}$ limitation of the SCR $(1000 \mathrm{~A} / \mu \mathrm{s})$. The delay between initial switch trigger and the magnet current pulse also allows time to disable the Main Injector proton extraction kicker in case of failure of the power supply. The small saturating choke $\mathrm{L}_{\text {sch }}$ protects the SCR by limiting the initial $\mathrm{dI} / \mathrm{dt}$ of the thyristor switch to $100 \mathrm{~A} / \mu$ s for the first microsecond after turnon. To limit the reverse current of the thyristor, which has a relatively long recovery time, a stack of fast recovery diodes is connected in series. When the first saturating reactor L2 becomes conductive the energy from C1 transfers to the second stage capacitor C2. The second stage is located about $10 \mathrm{~m}$ away and connected to the first stage by a pair of coaxial cables (RG 220/U). Saturation of L3 initiates the discharge of C2 into the ringing circuit L4, C3. Capacitor C3 is connected to the sweep magnet (inductance L4) via the strip line.

After the magnet is energized, all the voltages ring at half the voltage across the sweep magnet because the secondary circuit ground is at the center tap of the sweep magnet. Voltages calculated for the circuit with an initial charge on the energy storage capacitor of 3 $\mathrm{kV}$ are plotted in Figure 16.

The first stage switching reactor L2 is based on four $2.3 \mathrm{mV}$-sec/turn Magnetic Metals Square-50 $(25 \mu \mathrm{m})$ nickel-iron tape cores. The winding is split into two equal halves. Each half has 8 turns. The circuit between the step-up transformer and the reactor is balanced, with peak voltages of $\pm 15 \mathrm{kV}$. When the reactor saturates, the voltages on the two capacitor terminals approach $15 \mathrm{kV}$, and the voltage on one of the two output terminals approaches $30 \mathrm{kV}$. The other terminal remains at ground potential until the second reactor saturates. A DC bias current of up to $5 \mathrm{~A}$ is provided for both reactors L2 and L3.

The second stage reactor is based on three $3.0 \mathrm{mV}$-sec/turn Allied-Signal 2605-SC Metglas cores, annealed and cowound with 3- $\mu$ m mylar insulation. The reactor L3 has 4 turns. With these windings, the volt-seconds capability of the cores is $150 \mathrm{mV}$-sec for the first stage, and $36 \mathrm{mV}$-sec for the second stage. The minimal size and volt-seconds requirements on the cores is determined by the fact that if at any time both cores are simultaneously conducting, the final state of the core reset point is undetermined, leading to unacceptable jitter in the 
subsequent pulse timing. Inductive voltage per lamination in the first stage is about $0.6 \mathrm{~V}$, and in the second stage about $2.2 \mathrm{~V}$. Nickel-iron tape cores were chosen for the first stage because of their very square B-H loop, relatively large packing factor, acceptable losses and adequate insulation. Metglas cores were chosen for the second stage because of their small losses and good insulation properties of the mylar film.

The basic building block of the pulse compression circuit is the single-loop energy transfer circuit with two capacitors and an inductor. The inductor represents the saturated inductance of the switching reactor. The energy transfer efficiency of the simple circuit is $4 \mathrm{a} /(1+\mathrm{a})^{2}$ where $\mathrm{a}$ is the ratio of the value of the two capacitances, or $\mathrm{a}=\mathrm{C} 2 / \mathrm{C} 1$ for energy transfer from the first stage to the second stage. If the capacitor values are equal, the loop transfer efficiency is $100 \%$ in the absence of losses.

Measurements of the losses in energy transfer were made at $2.9 \mathrm{kV}$. The initial energy stored on capacitor $\mathrm{C} 0$ was $42 \mathrm{~J}$. Energy delivered to capacitor $\mathrm{C} 1$ was $35 \mathrm{~J}$. Of the $7 \mathrm{~J}$ loss in this energy transfer the losses were distributed $4 \mathrm{~J}$ to the SCR switch and $3 \mathrm{~J}$ to the balance of the circuit. Energy transferred from $\mathrm{C} 1$ to $\mathrm{C} 2$ was $31.5 \mathrm{~J}$. The losses were dominated by resistive losses in the windings. Switching losses in the cores, determined from the B-H loops, are small, $0.3 \mathrm{~J}$ in the first stage, and $0.5 \mathrm{~J}$ in the second stage. Finally energy transferred to the ringing circuit was $22 \mathrm{~J}$.

The least efficient part of the circuit is the final transfer from capacitor $\mathrm{C} 2$ to the ringing circuit L4, C3. When the reactor L3 saturates, a single current pulse passes to the ringing circuit. In addition to the normal resistive losses, it is relatively inefficient for two reasons.

First, it is necessary to prevent saturating the reactor a second time, and passing a second current pulse that effectively extracts energy from the magnet circuit, due to the ringing nature of the load. The voltage on capacitor C2 must be reverse biased by at least $40 \%$ of its maximum voltage immediately after the first current pulse. This is naturally accomplished in the circuit if the ratio of capacitor C3 to capacitor C2 is at least 2. For the energy-transfer loop, the ratio of the minimum voltage on the first capacitor after discharge to the initial charge voltage is $(1-a) /(1+a)$ where $a$ is the ratio of the value of the two capacitances, in this case $\mathrm{a}=\mathrm{C} 3 / \mathrm{C} 2$. The energy transfer efficiency of the circuit, $4 \mathrm{a} /(1+\mathrm{a})^{2}$, is about $89 \%$ for a capacitance ratio of 2 . This reflected voltage also provides a reset pulse to the reactors, ensuring highly stable operation even with no reverse bias current.

Second, with the addition of the inductance of the sweep magnet, L4, the final energy transfer circuit becomes a double-loop energy transfer circuit. The efficiency falls further for practical saturated inductance L3 because current is also flowing in the magnet circuit inductance L4. Energy-transfer efficiency after the single forward current pulse was calculated using the linear coupled-mesh equations for the circuit that includes C2, C3, L3, and L4. The voltage $v_{2}$ on capacitor C2 is given by

$$
v_{2}=a_{1} \cos \left(\omega_{1} t\right)+a_{2} \cos \left(\omega_{2} t\right)
$$

where 
$\omega_{1,2}^{2}=\frac{1}{2 L_{3} L_{4} C_{3} C_{4}}\left[L_{3} C_{3}+L_{4} C_{4}+L_{4} C_{3} \pm \sqrt{\left(L_{3} C_{3}+L_{4} C_{4}+L_{4} C_{3}\right)^{2}-4 L_{3} L_{4} C_{3} C_{4}}\right]$

and

$a_{1,2}= \pm \frac{1-L_{3} C_{3} \omega_{2,1}^{2}}{L_{3} C_{3}\left(\omega_{1}^{2}-\omega_{2}^{2}\right)}$

The transfer efficiency is determined by solving Eq. 3 for the voltage remaining on capacitor C2 after the initial positive current pulse. It is plotted in Figure 17 as a function of inductance L3 for $\mathrm{C} 2=0.1 \mu \mathrm{F}, \mathrm{C} 3=0.2 \mu \mathrm{F}$, and L4 $=0.324 \mu \mathrm{H}$ (L4 here includes the inductances of the sweep magnet and the strip line). For typical saturated inductance of the output switch, about $0.5 \mu \mathrm{H}$, this efficiency is about $80 \%$.

The power supply was built and tested for 7 million pulses at $3 \mathrm{kV}$ (full charge). At $3 \mathrm{kV}$ charge, the output current amplitude is $11.6 \mathrm{kA}$. Oscilloscope pictures of voltages and currents are shown in Figures 18 and 19.

\section{CONCLUSIONS}

1. The sweeping system magnet and power supply have been built and successfully tested.

2. A sweep radius of up to $0.5 \mathrm{~mm}$, corresponding to a peak sweep magnet current amplitude of over $10 \mathrm{kA}$, is easily achievable with the existing circuit. Simple modifications to the circuit may allow for additional future increase in the peak current.

3. The magnetic field distribution in the sweep magnet is satisfactory and confirms the design predictions.

4. The power supply works as predicted and is reliable. Investigation into core materials has led to a choice of reliable core materials for the power supply. The power supply jitter does not exceed \pm 2 ns, while slow drift is about $20 \mathrm{~ns}$. The total number of test pulses to date at full voltage is over 7 million. 


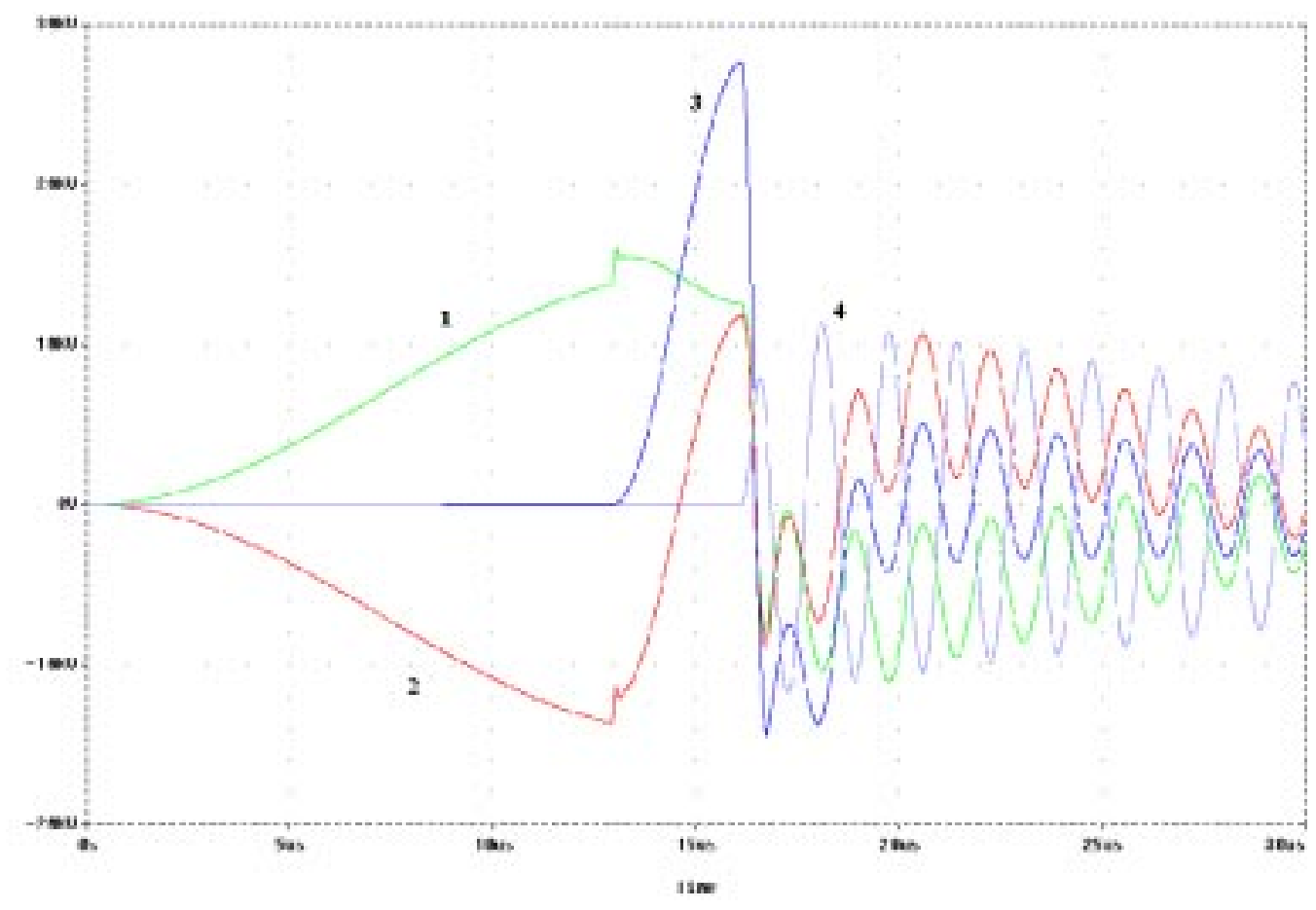

Figure 16. SPICE model voltage waveforms. 1- First stage capacitor C1 voltage, positive side, 2 - capacitor C1 voltage, negative side, 3 - second stage capacitor C2 voltage, 4 - ringing circuit capacitor $\mathrm{C} 3$ voltage.

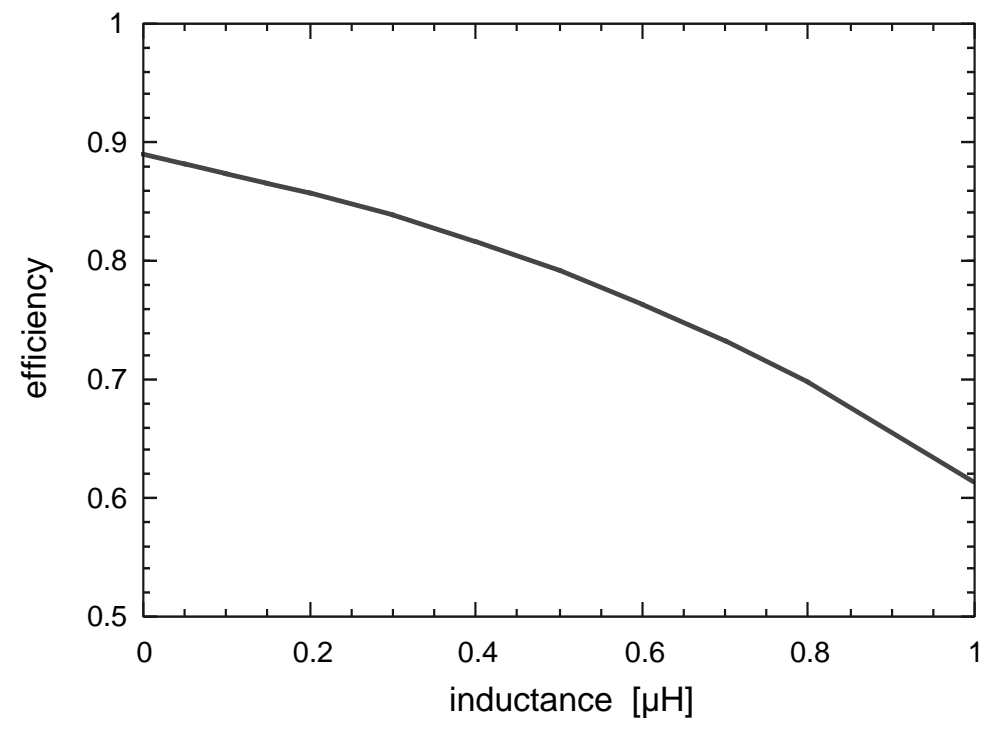

Figure 17. Theoretical efficiency of energy transfer from capacitor $\mathrm{C} 2$ to the ringing sweep magnet circuit through the saturated inductance of the output switch L3. 


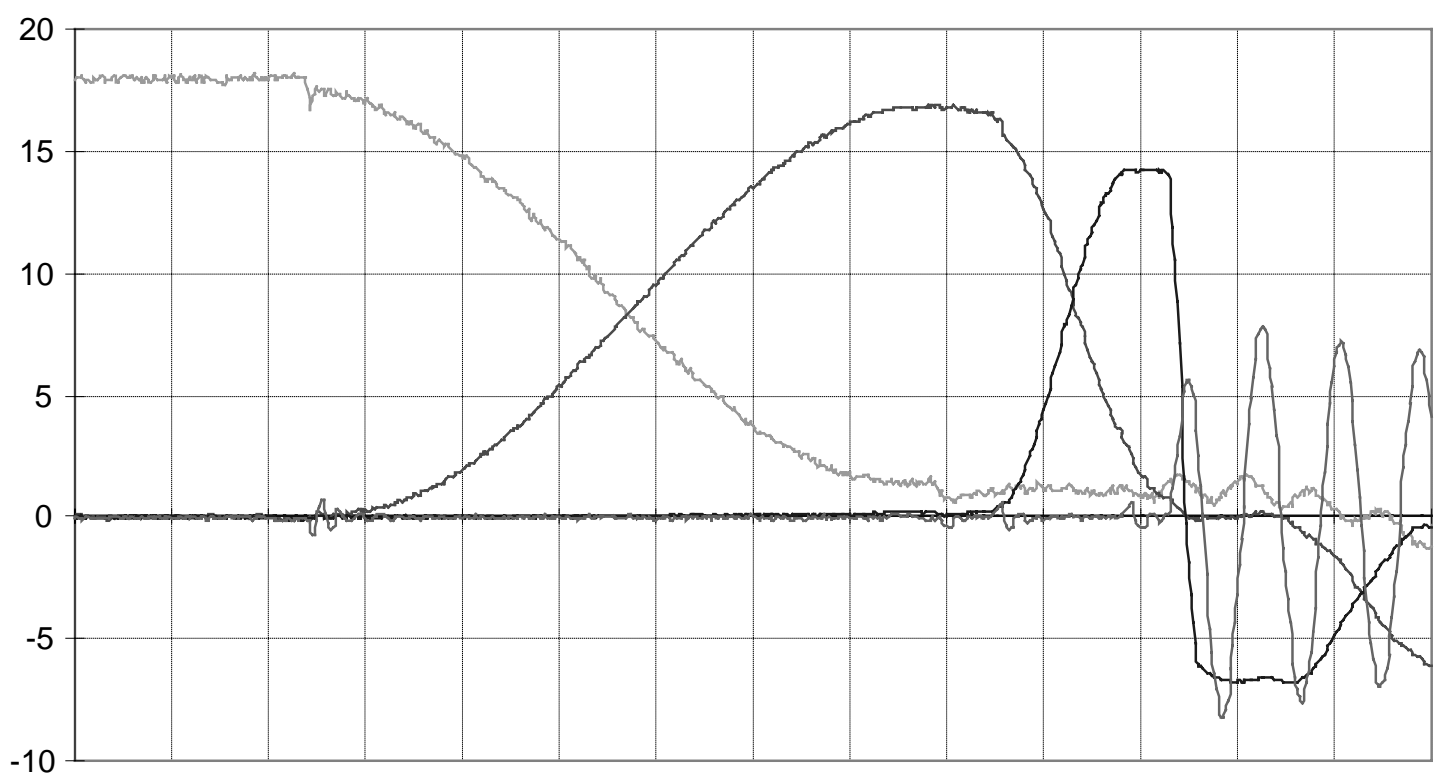

Fig. 18. Scope picture of voltages in compression stages. Initial voltage $=1.8 \mathrm{kV} .1$ first charging capacitor x 10. 2 - first compression stage capacitor. 3 - second compression stage capacitor. 4 - ringing circuit (magnet) voltage.

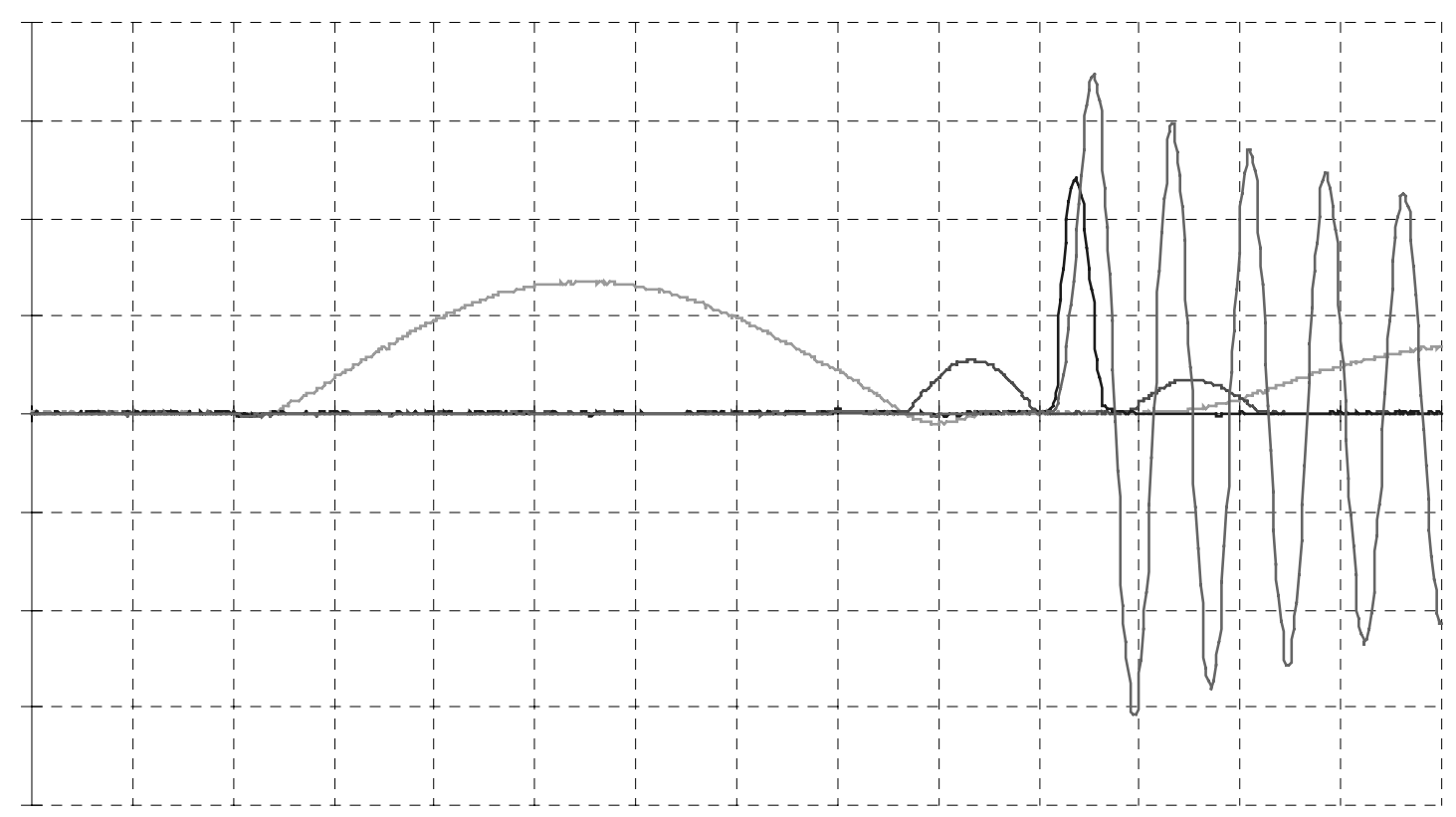

Fig. 19. Scope picture of currents in compression stages of power supply. Peak magnet current is $7 \mathrm{kA}$, corresponding to $2.2 \mathrm{kV}$. Vertical scale $=1 \mathrm{kA} /$ div. Horizontal scale $=2$ $\mu$ s/div. 1 - SCR current. 2 - first compression stage. 3 - second compression stage. 4 ringing circuit (magnet) current. 
*Present address: Lawrence Berkeley National Laboratory, Berkeley, CA 94720

$\dagger$ Present address: Dept. of Mechanical Engineering, Stanford University, Stanford, CA 94305

[1]. F. M. Bieniosek, A beam sweeping system for the Fermilab antiproton production target, Fermilab-TM-1857 (August 1993).

[2] P.P. Bagley, et. al., Summary of the TeV33 Working Group ,in 1996 Snowmass Conf.

[3] C. M. Bhat, N. V. Mokhov, Calculation of Beam Sweeping Effect for the Fermilab Antiproton Source, Fermilab-TM-1585 (1989).

[4] S. C. O'Day and F. M. Bieniosek, $8.9 \mathrm{GeV}$ pbar yield measurements at the Fermilab antiproton source, Nuclear Instrum. and Methods A343, p. 343-350 (1994).

[5] C. Hojvat and A. Van Ginneken, Calculation of antiproton yields for the Fermilab antiproton source, Nuclear Instr. and Methods 206, p. 67 (1983).

[6]. F. M. Bieniosek, Summary of results from the beam sweep test module, Fermilab Internal Pbar Note \#556, 1 Jun 1995.

[7] F. M. Bieniosek, Beam-Sweep Magnet with Rotating Dipole Field, Fermilab Internal Pbar Note\#561 (1996).

[8] C. W. Chen, Magnetism and Metallurgy of Soft Magnetic Materials (Dover, 1977), p. 474. 